\title{
Cardiovascular Aspects of Coronavirus Disease-2019
}

\author{
Refli Hasan* \\ Department of Internal Medicine, Faculty of Medicine, Universitas Sumatera Utara, Medan, Indonesia
}

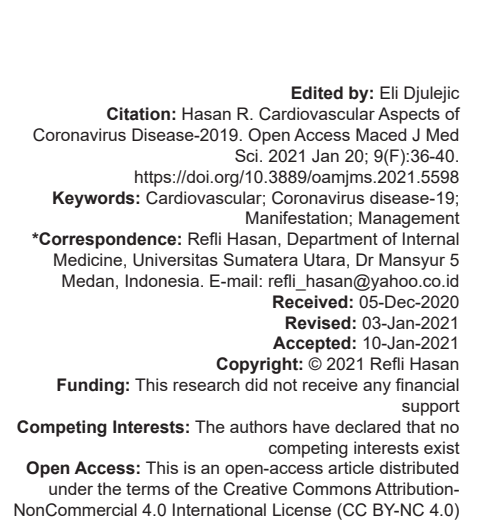

Introduction

Since the first case of coronavirus disease-2019 (COVID-19) was reported in China in December 2019, the disease has spread throughout the world and becomes a pandemic [1], [2], [3], [4], [5], [6].

The World Health Organization (WHO) declared COVID-19 as a pandemic on March 11, 2020 [2], [7], [8], [9], [10]. COVID-19 has influenced the world's medical, psychological, and socioeconomic aspects. It is probably the greatest global thread in the $21^{\text {st }}$ century [9], [11], [12], [13]. The disease is caused by a novel positive-sense singlestranded ribonucleic acid (RNA) betacoronavirus which is severe acute respiratory syndrome coronavirus (SARS-CoV)-2 [2], [3], [14], [15], [16]. SARS-CoV and SARS-CoV-2 share $79.6 \%$ of their genomic sequence [8], [12], [13]. The virus belongs to the Coronaviridae family which is thought to be descended from bat before it can infect human [2], [3], [14], [15], [16]. The transmission from bat to human is suspected through intermediate reservoirs such as civet [15]. This is not the first time in which a coronavirus infects human. Previously, two species of coronavirus have caused epidemic: SARS-CoV in 2002 and Middle Eastern respiratory syndrome-coronavirus (MERS-CoV) in 2012 [8], [9], [10]. Until the $1^{\text {st }}$ week of November
2020 , a total of $47,362,304$ cases have been reported globally with $1,211,986$ deaths [17].

COVID-19 may manifest as respiratory and gastrointestinal symptoms such as dry cough, shortness of breath, abdominal pain, nausea, and diarrhea, but later cardiovascular manifestations have been reported [1], [3], [16]. It is not surprising since previous epidemics of coronavirus possessed symptoms of myocarditis [1]. The symptoms are usually mild (81\%). Only $14 \%$ progress to severely ill and $5 \%$ develop critical illness [15], [18]. Cardiovascular disorders and COVID-19 are connected in reciprocal fashion [2], [19]. The proportion of COVID-19 patients with underlying cardiovascular disease is higher compared to the proportion of patients with other or without any comorbidities [2], [7]. A study in China reported that $29 \%$ patients had myocardial injury while another study reported a rate of $20 \%$ patients with myocardial injury [1]. Another clinical data reported that $15-30 \%$ patients with COVID-19 had hypertension and $2.5-15 \%$ had coronary heart disease [14]. In the other hand, patients with underlying cardiovascular injury have higher risk for cardiovascular events from COVID-19 [3]. Patients with underlying cardiovascular diseases tend to have more severe symptoms of COVID-19. In result, this will cause more severe complications and higher mortality rate [16], [18], [20]. We will discuss about the cardiovascular aspects of COVID-19. 


\section{Pathogenesis of Cardiovascular Involvement in COVID-19}

COVID-19 spreads mainly through respiratory droplets but several evidences have proven that fecooral transmission is possible [8], [15], [16]. Its median incubation period is $4-5$ days [8]. The SARS-CoV-2 has spike (S) protein in its envelope surface. The protein is important for viral invasion. This $S$ protein will bind with $\mathrm{S} 1$ subunit of angiotensin converting enzyme (ACE) 2 which expressed in human cells, initiating viral invasion. The virus then enters the cell with the aid of transmembrane serine protease (TMPRSS) 2 [1], [3], [7], [8], [14], [15], [18]. Both ACE2 and TMPRSS2 must be expressed in the same cell to initiate infection [9]. After entering the host cell, viral mRNA is translated to produce viral proteins. The proteins are then cleaved and assembled to form new viruses. New viruses fuse with plasma membrane and are released into extracellular space, ready to invade other cells [15]. SARS-CoV-2 affinity with ACE2 is stronger than other SARS-CoV [9], [19], [18].

ACE2 is mainly expressed in type I and II lung alveolar epithelium, brain, liver, kidney, pericytes, cardiomyocytes, enterocytes in the small intestine, and endothelial cells. This data support the theory of viral invasion into cardiomyocyte [1], [3], [14], [16], [19], [18]. Invasion may be occur from spreading through bloodstream [14]. More than $7.5 \%$ myocardiums express ACE2 which facilitate direct cardiac injury [10], [16], [18]. However, cardiovascular injury in COVID-19 is multifactorial [18]. Invasion will trigger immune response and inflammation and in the end result with myocardial necrosis. Within a few days, the necrosis will cause contractility disturbance and other clinical manifestations [1], [7], [14], leading to increased level of myocardialbiomarkers,abnormalitiesinelectrocardiography,and echocardiography [1], [3], [14]. Inflammation will trigger cytokines release, particularly interleukin-6, and cause damage in cardiomyocytes. Cardiovascular damage from inflammation is higher in hospitalized patients with severe or critical COVID-19 [1], [3]. Excessive production of cytokines results in abnormal inflammatory response called cytokine storm and is suspected to be a culprit of cardiovascular manifestations in COVID-19 patients [1], [3], [7], [10], [11], [12], [13], [14], [15], [20]. Inflammationalsoincreasestheoccurrenceofintravascular coagulopathy along with the risk of thrombosis in coronary vessels [1], [3], [8], [9], [10], [11], [12], [14], [15].

On the other hand, autoantibody is also involved in the pathogenesis of cardiovascular manifestations. There is a molecular mimicry between SARS-CoV-2 protein and S2 regions of cardiac myosin [1]. SARS-CoV-2 invasion can also downregulate ACE2 expression. ACE2 acts as protective agent toward fibrosis in cardiomyocytes.
Therefore, its downregulation will worsen the cardiovascular damage [3], [10], [14], [19]. Pulmonary dysfunction in COVID-19 triggers hypoxemia and insufficient oxygen supply to many organs including the heart. Furthermore, myocardial oxygen demand is increased due to inflammation. This situation creates ischemic injury of myocardium and its consequences [3], [7], [8], [10], [14], [15], [16], [17].

\section{Cardiovascular Manifestations of COVID-19}

Approximately $\quad 20-30 \%$ of hospitalized COVID-19 patients showed cardiac complications. Some patients even develop cardiovascular manifestations despite the absence of respiratory manifestations [15]. In general, patients with cardiovascular injuries had advanced age and more comorbidities [1], [3], [9], [18]. Elder population and the presence of comorbidities significantly increase the risk of developing severe COVID-19 [15], [19]. This may be caused by degenerative impairment of immune system in older individuals [2], [7], [9], [18], in contrast with children who have stronger innate immunity, fewer comorbidities, differences in maturation of viral receptors, and prior exposure to other coronavirus species [2]. Cardiovascular manifestations of COVID-19 are clinical heart failure, chest pain, acute coronary syndrome (ACS), and arrhythmias [1], [2], [3], [14], [15], [20].

ACS is one of initial cardiovascular manifestations in COVID-19, marked by ST segment elevation from electrocardiogram. ACS is suspected to be caused by plaque rupture, coronary spasm, and microthrombi formation. This phenomenon is reported by several case series [18], [19], [20]. Rhythm disturbance is more prominent in patients with underlying abnormalities of cardiac structure and function [1], [3]. Arrhythmia in COVID-19 is caused by direct myocardial infection and activated sympathetic nervous system [9], [18], [19], [20]. On the other hand, arrhythmia may be caused by side effect of medications used for COVID-19 [19]. Arrhythmia may occur solely without any other signs and symptoms such as fever or cough [7], [19]. Arrhythmia is observed in $16.7 \%$ of COVID-19 patients and in $44 \%$ of patients in Intensive Care Unit [9], [19]. Palpitation following arrhythmia was found in $7.3 \%$ COVID-19 patients in Wuhan [2], [7], [8], [19], [18]. The types of arrhythmia include atrial fibrillation, conduction block, ventricular tachycardia, and ventricular fibrillation [9], [19]. Malignant arrhythmia is more common in patients with elevated cardiac troponin level [2], [7], [8], [19], [18]. Heart failure occurs in $23 \%$ patients while myocardial injury (myocarditis and myocardial infarction) is reported in 7.2$17 \%$ patients. Heart failure, myocarditis, and myocardial infarction are the results of systemic inflammation 
and hypoxia [9], [19]. Coagulation abnormality even disseminated intravascular coagulation is reported in COVID-19 patients who may increase the risk of thromboembolic events. If occurred in cardiovascular system, this event will manifest as several signs and symptoms related to ACS and heart failure [2], [19].

\section{Diagnosis of Cardiovascular Disorders Related to COVID-19}

The standard diagnosis of COVID-19 requires specimen's collection from the upper or lower respiratory tract for real time polymerase chain reaction (RT-PCR) assay [3], [15]. The preferred specimens for RT-PCR are nasopharyngeal swab, followed by feces [15]. Supporting evaluation showed decreased total white blood cell and lymphocyte count with increased neutrophil to lymphocyte ratio [3], [15]. SARSCoV-2 specific antibodies may support the diagnosis. Immunoglobulin $\mathrm{M}$ ( $\mathrm{lgM}$ ) and immunoglobulin $\mathrm{G}$ ( $\mathrm{lg} \mathrm{G}$ ) are detected as early as $2-4$ days after symptoms. The antibodies gradually increase during the first 3 weeks after onset of disease [15].

Myocardial damage may be detected from electrocardiographic changes, radiologic imaging such as cardiacmagnetic resonance imaging, and biomarkers level such as troponin, creatine kinase, lactate dehydrogenase, and creatine kinase MB isozyme [1], [2], [3], [8], [9], [14]. A meta-analysis reported that cardiac troponin I levels were abnormal in 8-12\% hospitalized patients with COVID-19 [1]. Echocardiography, angiography, and other cardiac imaging are not routinely done in patients with hemodynamically stable and moderate elevation of troponin. All procedures should be conducted prudently by considering risk and benefit for patients and healthcare personnel [1], [9]. Postmortem cardiac biopsy may be conducted to demonstrate infiltration of inflammatory cells, moderate pericardial effusion with light yellow and clear liquid and mild edema, suggesting pericardial inflammation involvement in the cardiovascular disorder [1], [3], [8]. However, this examination is too invasive; therefore, non-invasive investigations and cardiac biomarker examinations should be maximized in diagnosing cardiovascular events in patients with COVID-19 [1]. Thrombogenesis is increased marked by elevated D-dimer [3], [20].

\section{Management of Cardiovascular Disorders Related to COVID-19}

Until present, there is no specific treatment for COVID-19 [18], [19]. There have been several ways either to prevent the spread or to slow the progression of the disease for those who have been exposed with SARS-CoV-2.

Preventing SARS-CoV-2 exposure is the main effort to slow the progression of COVID-19 spreading. Isolation is still an excellent choice to prevent the spreading of the virus SARS-CoV-2. However, complete isolation is not always available to be done since socioeconomic activity cannot be stopped completely.

Vaccination is also one of the ways to promote immunity against the spread of SARS-CoV-2. Vaccination has become the most promising approach to control this pandemic. At present, the vaccine is being developed and tested for its efficacy and safety [15].

As treatment priority, the elderly population with underlying comorbidities should always be the priority in the management of COVID-19 [2], [3], [10].

The use of antivirals has long been proposed as the choice of treatment for COVID19. Several antivirals such as remdesivir, ribavirin, lopinavir, and ritonavir are used empirically in the management of COVID-19. These antivirals prevent invasion by interfering viral RNA synthesis. Early administration of antiviral and utilization of combined antivirals are beneficial for alleviating clinical course [2], [7], [10], [15], [16], [18]. Another treatment option such as immunosuppressant has been found used as COVID-19 in patients with myocarditis but its utilization in the virus itself still needs more investigations [1]. A candidate from this class of drug is tocilizumab [18]. Hydroxychloroquine also showed positive effect in COVID-19 based on several studies. It acts by preventing endosomal acidification, viral internalization, and modulate immune. Another class of drugs used in management of COVID-19 is antivirus and anti-inflammation drugs, such as azithromycin and steroids. Azithromycin has positive effect as antivirus and anti-inflammation. Steroids are used to alleviate symptoms associated with cytokine storm [7], [10], [16], [18]. Unfortunately, the use of hydroxychloroquine, azythromycin, and antivirals together would increase the risk of QT prolongation and torsades de pointes [1], [2], [3], [7], [8], [10], [15], [18].

Intravenous IG is also being investigated as one of treatment options for COVID-19. This treatment acts by modulating patient's immune response toward viruses [15].

Recently, convalescent plasma has also been used to manage COVID-19 [1], [3], [15]. Anticoagulant, particularly heparin, is also considered for COVID-19 management since the disease creates a state of hypercoagulability[15],[16],[18]. Extracorporeal membrane oxygenation supports patient's cardiopulmonary function during critical period of disease [16], [18].

Of all the comorbidities may be found in COVID-19 patients, the management of COVID-19 
patients with cardiovascular complications is mainly supportive due to some conditions found in patients with cardiovascular conditions. Discontinuation of ACE inhibitor or angiotensin receptor blocker is not recommended as the drugs may increase ACE2 expression and give protective effect after ACE2 downregulation from SARS-CoV-2 invasion [2], [3], [10]. Several studies also failed to prove that ACE inhibitors would facilitate the invasion of SARS-CoV-2 into host cells [7], [8], [14], [16], [18], [19].

\section{Outcome of COVID-19 Patients with Cardiovascular Manifestations}

COVID-19 patients with cardiovascular manifestation have poorer outcome [3], [7], [15], [18]. Higher cardiac troponin I level was associated with more severe disease outcome [1], [3], [14], [18], [19], [20]. Only 1\% myocardial injury was reported in COVID-19 survivors compared to $59 \%$ in deceased patients [1]. Patients with cardiovascular manifestations of COVID-19 should be routinely followed up every 1-3 months. Follow-up consists of history taking and physical examination, electrocardiography, and echocardiography [9]. They are also advised to avoid competitive sport or aerobic activity for 3-6 months until the resolution of myocarditis is confirmed [1].

\section{Conclusion}

COVID-19 not only affects respiratory system but also cardiovascular system. Several mechanisms underlie the cardiovascular involvement including direct invasion, inflammation, thrombosis, autoantibody, and imbalance of oxygen supply and demand. Cardiovascular manifestations of COVID-19 are ACS, arrhythmia, and heart failure. Therefore, beside routine examinations, auxiliary cardiovascular examinations such as cardiac biomarkers, cardiac imaging, electrocardiography, echocardiography, and angiography are considered in patients with cardiovascular manifestations. Similar with other patients, the management is mainly supportive. Antivirals, hydroxychloroquine, azithromycin, and steroid are empirically administered. Immunomodulators and convalescent plasma are being investigated for their effectiveness. Heparin is utilized to overcome coagulopathy. Routine follow-up is urged for patients who have recovered from COVID-19 with cardiovascular manifestations.

\section{References}

1. Hendren NS, Drazner MH, Bozkurt B, Cooper LT Jr. Description and proposed management of the acute COVID-19 cardiovascular syndrome. Circulation. 2020;141(23):1903-14. https://doi.org/10.1161/circulationaha.120.047349

PMid:32297796

2. Driggin E, Madhavan MV, Bikdeli B, Chuich T, Laracy J, BiondiZoccai G, et al. Cardiovascular considerations for patients, health care workers, and health systems during the COVID-19 pandemic. J Am Coll Cardiol. 2020;75(18):2352-71. https://doi. org/10.1016/j.jacc.2020.03.031

PMid:32201335

3. Geng Y, Wei Z, Qian H, Huang J, Lodato R, Castriotta RJ. Pathophysiological characteristics and therapeutic approaches for pulmonary injury and cardiovascular complications of Coronavirus disease 2019. Cardiovasc Pathol. 2020;47:107228. https://doi.org/10.1016/j.carpath.2020.107228

PMid:32375085

4. Ge H, Wang X, Yuan X, Xiao G, Wang C, Deng T, et al. The epidemiology and clinical information about COVID-19. Eur J Clin Microbiol Infect Dis. 2020;39(6):1011-9. https://doi. org/10.1007/s10096-020-03874-z

PMid:32291542

5. Ruslie RH, Darmadi D. Coronavirus disease-19 and mental health. Open Access Maced J Med Sci. 2020;8:268-71. https:// doi.org/10.3889/oamjms.2020.5304

6. Siregar GA, Siregar GP, Darmadi D, Ruslie RH. Coronavirus disease-19 and liver injury. Open Access Maced J Med Sci. 2020;8:154-7. https://doi.org/10.3889/oamjms.2020.5028

7. Zhao $\mathrm{M}$, Wang $\mathrm{M}$, Zhang J, Ye J, Xu Y, Wang $Z$, et al Advances in the relationship between Coronavirus infection and cardiovascular disease. Biomed Pharmacother. 2020;127:110230. https://doi.org/10.1016/j.biopha.2020.110230 PMid:32428835

8. Srivastava K.Association between COVID-19 and Cardiovascular disease. Int J Cardiol Heart Vasc. 2020;29:100583.

PMid:32715080

9. Guzik TJ, Mohiddin SA, Dimarco A, Patel V, Savvatis K, MareliBerg FM, et al. COVID-19 and the cardiovascular system: Implications for risk assessment, diagnosis, and treatment options. Cardiovasc Res. 2020;116(10):1666-87.

PMid:32352535

10. Lazaridis C, Vlachogiannis NI, Bakogiannis C, Spyridopoulos I, Stamatelopoulos K, Kanakakis I, et al. Involvement of cardiovascular system as the critical point in Coronavirus disease 2019 (COVID-19) prognosis and recovery. Hellenic J Cardiol. 2020; Hellenic J Cardiol. 2020; 2020:1-15. https://doi. org/10.1016/j.hjc.2020.05.004

PMid:32534109

11. Siregar GA, Siregar GP, Darmadi D. Gastrointestinal aspects of COVID-19: A review. Open Access Maced J Med Sci. 2020;8:524. https://doi.org/10.3889/oamjms.2020.4891

12. Ruslie RH, Darmadi D, Siregar GA. Pediatric immunization practice during Coronavirus disease-2019 pandemic. Open Access Maced J Med Sci. 2020;8:258-60. https://doi. org/10.3889/oamjms.2020.5125

13. Ruslie RH, Darmadi D, Siregar GA. Susceptibility of Coronavirus disease-19 in pediatric population. Open Access Maced J Med Sci. 2020;8:363-5. https://doi.org/10.3889/oamjms.2020.5240

14. Hafiane A. SARS-CoV-2 and the cardiovascular system. Clin Chim Acta. 2020;510:311-6.

PMid:32681935 
15. de Vries AA. SARS-CoV-2/COVID-19: A primer for cardiologists. Neth Heart J. 2020;28(7-8):366-83. https://doi.org/10.1007/ s12471-020-01475-1

PMid:32671650

16. Tan W, Aboulhosn J. The cardiovascular burden of Coronavirus disease 2019 (COVID-19) with a focus on congenital heart disease. Int J Cardiol. 2020;309:70-7. https://doi.org/10.1016/j. ijcard.2020.03.063

PMid:32248966

17. World Health Organization. Coronavirus Disease (COVID-19) Outbreak Situation. Geneva: World Health Organization; 2020. Available from: https://www.covid19.who.int. [Last accessed on 2020 Nov 05].

18. Kang $Y$, Chen T, Mui D, Ferrari V, Jagasia D, ScherrerCrosbie $\mathrm{M}$, et al. Cardiovascular manifestations and treatment considerations in COVID-19. Heart. 2020;106(15):1132-41. https://doi.org/10.1136/heartjnl-2020-317056

PMid:32354800

19. Nishiga M, Wang DW, Han Y, Lewis DB, Wu JC. COVID-19 and cardiovascular disease: From basic mechanisms to clinical perspectives. Nat Rev Cardiol. 2020;17(9):543-58. https://doi. org/10.1038/s41569-020-0413-9

PMid:32690910

20. Wu L, O'Kane AM, Peng H, Bi Y, Motriuk-Smith D, Ren J. SARSCoV-2 and cardiovascular complications: From molecular mechanisms to pharmaceutical management. Biochem Pharmacol. 2020;178:114114. https://doi.org/10.1016/j. bcp. 2020.114114

PMid:32579957 